Results As part of Hampshire's Dementia Friendly Communities initiative and Sway parish council's objectives; business in Sway are becoming officially Dementia Friendly through attendance at Dementia Friends sessions and always having a dementia friendly helper on duty. There is partnership working between the parish council, other local charities, volunteer groups and the dementia action group.

Conclusion Dementia is a progressive terminal disease which benefits from a palliative care approach at all stages. Palliative care does not only have to be provided by health services or by adults. It can also be delivered by neighbours and community partners of all ages. Harnessing the experiences and passion of a community including teenagers (with school or vocational curriculums to meet) has the potential to support Dementia Friendly Communities where people live and die well with dementia.

\section{P-141 HOSPICE PHILOSOPHY IN DEMENTIA CARE: TAILORING INDIVIDUALISED TRAINING}

\footnotetext{
1,2 Helen Reeves, ${ }^{3,4}$ Angela Causton, ${ }^{3,4}$ Sophie Meredith, ${ }^{2}$ Joanne Jinks, ${ }^{5}$ Davina Lytton, ${ }^{6,7}$ Michael Hurt. ${ }^{1}$ St Giles Hospice, Whittington, UK; ${ }^{2}$ St Giles Hospice, Walsall, UK; ${ }^{3}$ Accord Group; ${ }^{4}$ Pathways 4 Life; ${ }^{5}$ Age UK, Walsall; ${ }^{6}$ Walsall Council; ${ }^{7}$ Walsall CCG
}

\subsection{6/bmjspcare-2016-001245.164}

In June 2015 a partnership was formed in a health economy in the West Midlands between a hospice, CCG, council and a housing, health and social care organisation with the aim being to improve dementia and end-of-life care in care homes for a 12month pilot. The hospice and housing provider each provided a dementia support worker (DSW) with the focus of their roles being to help support, educate and signpost care homes within the borough to improve quality of life for people living with dementia.

The uniqueness of this pilot project has been the joint leadership and management between the two providers commissioned. Both have combined their knowledge and expertise to provide a seamless service to people living with dementia in care homes.

Within the initial 12 month pilot the DSWs have managed to make vast improvements. Within the first 12 months they have supported 33 out of 56 care homes providing individualised, bespoke support, training and advice to each home. They have also enabled 11 care homes to be part of the Activity Coordinator Forum, created by the DSW service; 8 care homes are now implementing elements of the Namaste Care Programme; three care homes have implemented Advance Care Plans as a result of the service and seven care homes have implemented the use of Pain Scales as a result of the service.

In addition to this there have been awards presented by the mayoress to celebrate four homes' engagement with the dementia support workers and also they have arranged for a dementia steering group to be formed that covers all professions and settings in the borough to ensure dementia patients get the same level of care, regardless of setting. It has been so successful it has been commissioned for a further 12 months with the focus being on improving dementia and end of life in the community.

\section{P-142 BREATHE BETTER - FEEL GOOD, DO MORE}

Lisa Wright, Stephen Oxberry, Michael Crowther . Kirkwood Hospice, Huddersfield, UK

10.1136/bmjspcare-2016-001245.165
Breathe Better, a palliative chronic lung disease program run over five sessions within the hospice support and therapy centre, was devised as a collaborative initiative between the hospice team, the acute trust, community services and CCG, providing information and practical support signposting patients to hospice services and reducing avoidable hospital admissions. Allowing patients to access hospice services and improve symptom management in end stage disease, changes patients' perceptions and helps to improve quality of life and self-management.

Patients are referred to the course via healthcare professionals, have a Medical Research Council Dyspnoea Score of 4 or 5, and are invited to attend with carers.

Over the five sessions goal setting is a common theme and continually revisited reinforcing positive achievements and support when required. Sessions include; Medication management, Relaxation and anxiety, Care planning and Fatigue management. Exercises are also completed during each session in a controlled and safe environment.

From feedback, patients feel more confident in self-management and ability to perform their daily activities. There has been a significant increase in patients accessing hospice services and recorded future care planning. Patients are achieving their goals and carers report seeing positive differences. Timely input and support is also being given to patients as their condition deteriorates. Importantly, hospital admissions for the six months post Breathe Better are less than half than the six months pre- Breathe Better, at a time when arguably more admissions would be expected.

The programme demonstrates that hospices are perfectly placed to address unmet palliative needs for patients with end stage lung disease. We have the skills, expertise and environment to begin to address end-of-life issues with patients and carers and this project demonstrate the huge benefits hospice care can offer this patient group.

\section{P-143 CLAN CLUB... CANCER, LEADING A NORMAL LIFE}

Myfanwy Howells. St Lukes' Hospice, Corringham, UK

\subsection{6/bmjspcare-2016-001245.166}

The St Lukes' Hospice Information Service provides information and support for individuals affected by cancer/life limiting illness at any stage of their journey. The Clan Club is a support group for children/young people aged 16-19 years with a cancer diagnosis ranging from diagnosis into survivorship and beyond. It also offers support to siblings and parents of children aged under six. There is no support group identified in Essex for this group of people.

The group provides the youngsters with the opportunity to meet peers, get support from trained facilitators and more importantly have fun. There are indoor and outdoor activities available and the youngsters are encouraged to partake as they are able. In the summer months there is greater emphasis on being more physically active with the winter months being more suitable to board/electronic games and creative activities. From a Macmillan grant other activities are funded e.g. bowling.

The adults have access to support for themselves. The sharing of information, hints and tips as well as managing the mire of tertiary care whilst ultimately getting the 'best' for their child are key themes of discussion.

All the individuals that attend the group have the opportunity to be referred to hospice services. This can include counselling 
for children/young people and adults, complementary therapy as well as individualised carers' support and access to a menu of therapeutic groups.

There are age-related outcome-based evaluations in place to measure the effectiveness of the group. All service users are encouraged to feedback any issues that would improve their experience of the group.

There is no similar group in Essex to meet the support needs of this group of people. In essence the Clan Club is a warm, friendly group that values individuality, promotes empowerment, but most importantly provides a focus for families to meet.

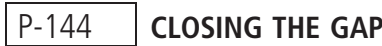

Manisha Cook, Caroline Whelldon. Hospice of St Francis, Berkhamsted, UK

\subsection{6/bmjspcare-2016-001245.167}

Background A retrospective study into the experiences of pulmonary fibrosis patients and carers identified holistic assessment and carer support as the main unmet needs of this client group. Pulmonary fibrosis is generally a rapidly progressive disease, causing great physical and psychological distress to those affected. The disease trajectory of Pulmonary fibrosis differs from Chronic Obstructive Pulmonary Disease (COPD) but often they are treated together in exercise and support groups.

With the aim of addressing the lack of specific pulmonary fibrosis support and to meet patient and carer needs a hospice based pulmonary fibrosis support group was established.

Method A twice monthly group was established within the Hospice of St Francis, led by a specialist palliative care physiotherapist with support from a palliative care nurse specialist. The sessions include a patient-led exercise programme and conclude with relaxation. Regular speakers join the group covering topics from future care planning, cognitive behavioural therapy, diet, fatigue management and social services.

Each patient completes a holistic needs assessment and we are commencing the use of Well-being star outcome measure tool to evaluate effectiveness. Carers are invited to complete a carer's support needs assessment tool (CSNAT).

Conclusion and future goals The group is still in its infancy. The aim is for sessions to be patient led with a nominated patient or carer as chairperson, treasurer etc.

We are engaging with local respiratory groups, lung clinics and Pulmonary Fibrosis charities to reach out to as many patients and carers within Hertfordshire and bordering counties and expand/ establish the group further. The most effective form of advertising has been through social media.

Carer focus is developing with plans to host education and peer support sessions. Our aim is to arm carers with knowledge, build confidence in regards to symptom management and future care planning. HOSPICE-PALLIATIVE CARE AMONG TERMINAL CANCER PATIENTS IN KOREA, 2008-2014

1,2So Jung Park, 1,3Yoon Jung Chang, 'Ju Yeon Bak, 'Eun Jung Park, ' ${ }^{1}$ Hyun Jung Jho, ${ }^{1}$ Jin Young Choi, 'Eun Mi Ahn, ${ }^{1,3}$ Yeol Kim. ${ }^{1}$ Palliative Care Clinic, National Cancer Centre, Republic of Korea; ${ }^{2}$ Department of Family Medicine, Graduate School of Yonsei University, Seoul, Republic of Korea; ${ }^{3}$ Department of Cancer Control and Policy, Graduate School of Cancer Science and Policy, Republic of Korea

\subsection{6/bmjspcare-2016-001245.168}

Background Utilisation of hospice-palliative care (HPC) has increased steadily; however, little is known about trend or pattern of this use in Korea. We aimed to describe an overview of nationwide statistics on the utilisation of HPC service of terminal cancer patients in Korea.

Methods Data were collected through Korean Terminal Cancer Patient Information System from Hospice-Palliative Care Units (PCU) designated by Korea's Ministry of Health and Welfare from 2008 to 2014. Descriptive statistics were used for the analysis. Trends were examined by annual percentage change and Cochran-Armitage test.

Results 56,433 patients used PCU for seven years. In 2008, only 19 hospitals (total 282 beds) were designated as PCU for terminal cancer patients by Korean government. With gradual increases, there were 57 designated PCU (total 950 beds) in 2014. The utilisation rate of PCU by terminal cancer patients increased from $7.3 \%$ of all cancer death in 2008 to $13.8 \%$ in 2014 . The patients' mean age was $67.1 \pm 12.9$ years, and $57.3 \%$ were male. Lung cancer patients made up the largest percentage of PCU admission. Increasing trends were observed in patient's awareness of terminal status from $67.3 \%$ in 2010 to $76.1 \%$ in 2014 . Average length of stay was $23.3 \pm 27.0$ days and $73.9 \%$ of discharged patients faced death in PCU.

Conclusions The number of terminal cancer patients received hospice care has steadily increased over the past several years in Korea. It is necessary to develop a variety of services that enhance the quality of end of life care by monitoring of hospice utilisation.

\section{P-146 SHARED CARE LIVER PROJECT}

Virginia Campbell, Sharon Quinn. St Luke's Hospice, Basildon, UK

\subsection{6/bmispcare-2016-001245.169}

There is growing recognition that people with advanced liver disease (ALD) have limited access to palliative care, despite a high symptom, psychological and social burden. Many have little opportunity to do end-of-life planning and some are referred to palliative care services as late as two days before death

Early hospice referral for patients with ALD and their carers seems at best a national aspiration rather than standard best practice. 